\title{
Autoestima y desempeño laboral de las enfermeras del Hospital Daniel Alcides Carrión-Callao
}

Zoraida Judith Huamán Gutiérrez $\bigsqcup^{1}$ Victor Tarazona Miranda $\bigsqcup^{2}$ y Paulo César Olivares Taipe

Resumen: El objetivo fue determinar la relación entre autoestima y desempeño laboral de las enfermeras del "Hospital Daniel Alcides Carrión". El método corresponde a un estudio básico, de nivel correlaciónal, enfoque cuantitativo, diseño no experimental y transversal. La población se conformó por 289 enfermeras, habiendose elegido a 165 del total de forma no probabilística. La recolección de datos se realizó a través de dos instrumentos con un valor de confiabilidad de 0,922 y 0,965 respectivamente. Para el análisis bivariado se utilizó la prueba de Chi-Cuadrado aplicándose el análisis de correspondencia simple. Los resultados señalaron que existe relación significativa entre autoestima y desempeño laboral en las enfermeras del hospital Daniel Alcides Carrión. $\left(\chi^{2}=33,898 ; p<0,01\right)$, también se demostró que existe relación significativa entre autoestima y la competencia cognitiva $\left(\chi^{2}=24,883 ; p<0,01\right)$, igualmente entre autoestima y las competencias actitudinales/sociales $\left(\chi^{2}=25,739\right.$; $p<0,01)$ y entre la autoestima y la competencia técnica $\left(\chi^{2}=18,092 ; p<0,05\right)$. La investigación concluye que un buen nivel de autoestima mejora significativamente el desempeño laboral en las enfermeras del hospital Daniel Alcides Carrión

Palabras clave: Autoestima; desempeño laboral; relación.

\section{Self-esteem and work performance of nurses at Daniel Alcides Carrión-Callao Hospital}

\begin{abstract}
The objective was to determine the relationship between self-esteem and job performance of the nurses at the "Hospital Daniel Alcides Carrión". The method corresponds to a basic study, correlational level, quantitative approach, nonexperimental and cross-sectional design. The population was made up of 289 nurses, 165 of the total having been chosen in a non-probabilistic way. Data collection was done through two instruments with a reliability value of 0,922 and 0,965 respectively. For the bivariate analysis, the Chi-Square test was used, applying the simple correspondence analysis. The results indicated that there is a significant relationship between self-esteem and job performance in the nurses of the Daniel Alcides Carrión hospital. $\left(\chi^{2}=33,898 ; p<0,01\right)$, it was also shown that there is a significant relationship between self-esteem and cognitive competence $\left(\chi^{2}=24,883 ; p<0,01\right)$, also between self-esteem and attitudinal / social competences $\left(\chi^{2}=25,739 ; p<0,01\right)$ and between self-esteem and technical competence $\left(\chi^{2}=18,092 ; p<0,05\right)$. The study concludes that a good level of self-esteem significantly improves job performance in nurses at the Daniel Alcides Carrión hospital.
\end{abstract}

Keywords: Self-esteem; work performance; relationship

Recibido: 10/10/2020. Aceptado: 24/12/2020. Publicado online: 31/12/2020.

(c) Los autores. Este artículo es publicado por la Revista PESQUIMAT de la Facultad de Ciencias Matemáticas, Universidad Nacional Mayor de San Marcos. Este es un artículo de acceso abierto, distribuido bajo los términos de la licencia Creative Commons Atribucion-No Comercia-CompartirIgual 4.0 Internacional.(http://creativecommons.org/licenses/by-nc-sa/4.0/) que permite el uso no comercial, distribución y reproducción en cualquier medio, siempre que la obra original sea debidamente citada. Para uso comercial, por favor póngase en contacto con revistapesquimat.matematica@unmsm.edu.pe

${ }^{1}$ UNMSM, Facultad de Ciencias Matemáticas. e-mail: zhuamang@unmsm.edu.pe

${ }^{2}$ UNMSM, Facultad de Ciencias Matemáticas, e-mail: vtarazonam@unmsm.edu.pe

${ }^{3}$ UNMSM, Facultad de Ciencias Matemáticas, e-mail: polivarest@unmsm.edu.pe 


\section{Introducción}

La autoestima en el mundo laboral es un estado ideal para que los empleados logren desempeñarse al máximo manifestándose en la manera de interactuar y relaciónarse con las demás personas. Según La Organización Mundial de la Salud (2014), "El bienestar psicológico es valioso en la formación integral de las acciones en el ámbito laboral"(p. 20). En esa dirección los centros de labores deben promover intervenciones para mejorar en el personal su salud mental ya sí reducir la ansiedad, por ello es fundamental establecer programas de orientación, prevención y de intervención principalmente en estrategias educativas y de apoyo psicosocial. Según Coopersmith (2015), "Evaluación que el sujeto tiene sobre sí mismo, poniendo de manifiesto actitudes de aprobación o desaprobación, en relación con su propia capacidad"(p. 35). Para Chiavenato (2007), "Las nuevas tendencias de las organizaciones demandan un nuevo tratamiento al recurso humano, convirtiéndolos en el recurso más valioso de la organización"(p. 52). Según el autor, en Latinoamérica los cambios del entorno laboral se entienden como una supervaloración del capital humano que crea un elemento intangible e invisible en la organización.

El primer autor en emplear el término competencia fue Chomsky generalizándose el término a diferentes ámbitos como una serie de conocimientos, capacidades, destrezas y actitudes propias para llevar acabo desempeños específicos (Trujillano, 2012). La Competencia laboral, según Lladó, Sánchez y Navarro (2013) el termino denota "la capacidad para recabar, organizar y sistematizar en el contexto de trabajo"(p. 12). Por otro lado, Keith y Newstrom consideran que "Un desempeño armonioso, eficiente y de cumplimiento total de las funciones asignadas en el trabajo"(p. 23). En vista de lo señalado, la autoestima y el desempeño laboral constituyen variables estrechamente relaciónadas dada su importancia en la satisfacción del trabajador asociada a su desempeño dentro de una organización. Por tanto, no constituyen conceptos nuevos en este contexto relaciónado al trabajo y a la función que desarrolla el trabajador en los centros de trabajo ligados a lo cognitivo, lo actitudinal y a su desempeño técnico.

Bernaola (2008) señala que "una autoestima baja es sentirse inútil para la vida: equivocado, no con respecto a tal o cual asunto, sino equivocado consigo mismo"(p. 56). Por consiguiente, desarrollar la autoestima es desarrollar la convicción de que uno es competente para vivir y capaz de desarrollarse laboralmente, en este sentido, Rodríguez (2013) señaló "la vida debe enfrentarse con mayor confianza, benevolencia y optimismo, lo cual nos ayuda a alcanzar nuestras metas y experimentar la plenitud del valor del trabajo"(p. 23). En vista de ello, la autoestima permite ampliar nuestra capacidad para desempeñar nuestras responsabilidades personales y laborales permitiéndonos estar mejor preparados para afrontar las adversidades la desesperación o a la derrota. Según Becerra (2017), "el desarrollo de las interrelaciónes mejora la autoestima de los trabajadores haciéndolo sentirse apto y competente en su vida laboral"(p. 34). Por consiguiente, la autoestima se relacióna con el desempeño laboral a través de actitudes y convicciones que muestran las personas al momento de enfrentar desafíos o dificultades de tipo laboral, específicamente se afirma que la autoestima trata del sentimiento de valía hacia uno mismo con un gran determinismo en el desempeño laboral de las personas.

A través de esta investigación se determinó la relevancia y asociación de las variables autoestima y desempeño laboral. Así mismo, cabe señalar que las enfermeras fueron evaluadas de acuerdo con cada variable de estudio, las mismas que se relaciónan a través de los instrumentos que fueron debidamente elegidos y validados por expertos a fin de cumplir con la rigurosidad del método científico. Por tanto, el estudio contribuye a la ciencia con los nuevos conocimientos adquiridos los mismos que son profundizados y analizados desde la óptica y reflexión teórica de las teorías que sustentan la presente investigación. Machuca (2016) en su investigación determinó una relación entre el desempeño laboral y los estudios de especialización, Reynaga (2015) Las conclusiones del estudio señalaron una correlación entre la motivación y el desempeño laboral con un valor de significancia de 0,003 y un valor de correlación de 0,488 que indicó una relación 
positiva y moderada. Asimismo, se determinó relaciónes positivas entre las dimensiones intensidad y participación con el desempeño laboral. Mireyna (2012) concluyó que la capacitación profesional mejora en cierta medida el desempeño laboral y respecto a las variables sociológicas se determinó que no se relaciónan con la satisfacción laboral o el desempeño laboral en comparación con los factores higiénicos y motivacionales.

En el Perú, los servicios públicos se caracterizan por conformar un conjunto de prestaciones que buscan en esencia la igualdad y el bienestar social de la comunidad, en este sentido, la enfermería es un servicio público que contribuye a preservar la vida y salud de las personas, por ello esta labor desempeña actividades en instituciones de salud pública funcionando como establecimientos para la atención y asistencia en el cuidado de la salud de las personas y/o usuarios del servicio. De esta manera, el entorno laboral se relacióna con aspectos conductuales y de satisfacción que incide directamente en el rendimiento de los trabajadores. En vista de las consideraciones anteriores, el objetivo de la presente investigación fue determinar la relación que existe entre la autoestima y el desempeño laboral en las enfermeras del Hospital Daniel Alcides Carrión, Callao.

\section{Metodología}

El estudio es de tipo básica. De acuerdo con Soto (2015), "la investigación básica representa el aporte de un cuerpo organizado de conocimientos y recoge información de la realidad para validarlo con el conocimiento científico"(p. 45). El diseño de investigación fue descriptivo correlaciónal. Según Vara (2015), "los estudios descriptivos correlaciónales determinan el grado de asociación entre variables"(p. 57). Según lo expuesto, una correlación es una medida del grado en que dos variables se hallan presuntamente conexas o relaciónadas. Por consiguiente, un estudio correlaciónal pretende determinar si los individuos con una puntuación alta en una variable también tiene puntuación alta en una segunda variable.

Variable: Desempeño laboral

De acuerdo con Chiavenato (2007): la operacionalizacion del desempeño laboral es:

Tabla 1

Matriz de operacionalización de la variable desempeño laboral

\begin{tabular}{|c|c|c|c|c|}
\hline Variable & Dimensiones & Items & Escala & Índice \\
\hline \multirow{3}{*}{ Desempeño laboral } & $\begin{array}{l}Y_{1}: \text { Competencia } \\
\text { cognitiva }\end{array}$ & $1,2,3,4,5,6,7,8$ & \multirow{3}{*}{ Ordinal } & \multirow{3}{*}{$\begin{array}{l}\text { Nunca: } 0 \\
\text { A veces: } 1 \\
\text { Frecuentemente: } 2 \\
\text { Siempre: } 3\end{array}$} \\
\hline & $\begin{array}{l}Y_{2}: \text { Competencias actitudi- } \\
\text { nales y sociales }\end{array}$ & $9,10,11,12,13,14,15$ & & \\
\hline & $Y_{3}:$ Competencia técnica & $16,17,18,19,20,21$ & & \\
\hline
\end{tabular}




\section{Variable: Autoestima}

Según Coopersmith (2015): la operacionalización es

\section{Tabla 2}

Matriz de operacionalización de la variable autoestima

\begin{tabular}{|c|c|c|c|c|c|}
\hline Variable & Dimensiones & Indicadores & Escala & Índice & Items \\
\hline \multirow{3}{*}{ Autoestima } & $\begin{array}{l}X_{1}: \\
\text { Autoestima es si } \\
\text { mismo general }\end{array}$ & $\begin{array}{l}\text { 1. Las cosas no me } \\
\text { molestan } \\
\text { 3. Cambio } \\
\text { 4. Tomar decisiones } \\
\text { 7. Costumbre } \\
\text { 10 Rendirse } \\
\text { 12. Ser "Yo mismo" } \\
\text { 13. Confusión } \\
\text { 15. Opinión } \\
\text { 18. Ser simpático } \\
\text { 19. Decir las cosas } \\
\text { 23. Desaliento } \\
\text { 24. Ser otra persona } \\
\text { 25. Confianza }\end{array}$ & $\begin{array}{l}\text { Verdadero: } \\
\text { si coincide con } \\
\text { su forma de } \\
\text { pensar } \\
\text { Falso: } \\
\text { no coincide con } \\
\text { su forma de } \\
\text { pensar }\end{array}$ & $\begin{array}{l}\text { Puntaje: } \\
0 \text { y } 1\end{array}$ & $\begin{array}{l}13 \text { ítems } \\
1,3,4,7,10, \\
12,13,15,18, \\
19,23,24 \text { y } 25\end{array}$ \\
\hline & $\begin{array}{l}X_{2}: \\
\text { Autoestima en el } \\
\text { área social }\end{array}$ & $\begin{array}{l}\text { 2. Hablar frente al } \\
\text { grupo } \\
\text { 5. Ser divertido } \\
\text { 8. Ser popular } \\
\text { 14. Ideas } \\
\text { 17. Descontento } \\
\text { 21. Preferencias }\end{array}$ & & & $\begin{array}{l}6 \text { ítems } \\
2,5,8,14,17 \mathrm{y} \\
21\end{array}$ \\
\hline & $\begin{array}{l}X_{3}: \\
\text { Autoestima en el } \\
\text { área del hogar }\end{array}$ & $\begin{array}{l}\text { 6. Ser alterado } \\
\text { 9. Sentimientos } \\
\text { 11. Familia } \\
\text { 16. Irse de casa } \\
\text { 20. Comprensión } \\
\text { 22. Presión }\end{array}$ & & & $\begin{array}{l}6 \text { ítems } \\
6,9,11,16,20 \\
\text { у } 22\end{array}$ \\
\hline
\end{tabular}

Nota. Teoría de Coopersmith

El método de estudio fue hipotético deductivo. Según Tamayo (2012), "el método hipotético deductivo tiene varios pasos esenciales, la observación del fenómeno a estudiar, elaboración de una hipótesis para explicar el fenómeno de estudio, deducción de los resultados comprobando la verdad de los enunciados"(p. 89). De acuerdo a este procedimiento, se comprueban las hipótesis a partir de una reflexión racional y deductiva a partir de la extrapolación de los resultados a un carácter general aplicando una regla de inferencia. Asimismo, la investigación desarrolló un enfoque cuantitativo. Hernández, Fernández y Baptista (2014) señalaron que "las exploraciones de este enfoque abordan la problemática de la investigación de forma secuencial y demostrativa sobre la realidad observada" (p. 41).

Por tanto, "se formulan hipótesis basándose en el cálculo preciso y riguroso de la matemática con la intención de verificar las teorías expuestas"(Hernández et al., 2014, p. 40). En vista de lo señalado, la investigación desarrolló un enfoque cuantitativo, dado que su análisis y medición tomó como base las mediciones numéricas en cada una de las variables y dimensiones de las variables, realizandose un análisis descriptivo e inferencial partir de los datos acopiados en el estudio. La unidad de análisis fue una mujer que labora en el Hospital Daniel Alcides Carrión. En cuanto a los instrumentos se utilizaron dos cuestionarios debidamente valiados y confiables determinándose que la autoestima y el desempeño laboral tienen alta confiabilidad $(0,922$ y 
0,961). De ahí que, los instrumentos fueron aplicados a la muestra en el tercer trimestre del año en curso.

Se especifica que "la población denota una serie de especificaciones o características en la totalidad"(Hernández et al., 2014, p. 52). La población corresponde a un total de 289 enfermeras que laboran en el "Hospital Daniel Alcides Carrión". Por otra parte, "la muestra es un subgrupo de elementos de la población a estimar"(Hernández et al., 2014, p. 62).

$$
n=\frac{Z^{2} P Q N}{\varepsilon^{2} N-1+Z^{2} P Q}
$$

\section{Datos asumidos:}

$Z(1,96)$

$P(0,50)$ : Proporción de éxito.

$Q(0,50)$ : Proporción de fracaso $(Q=1-P)$.

$\varepsilon(0,05)$ : Tolerancia al error.

$N$ (289): población

$n$ : muestra

Por lo que

$$
n=\frac{1,96^{2} \times 0,5 \times 0,5 \times 289}{0,05^{2} \times(289-1)+1,96 \times 0,5 \times 0,5}=165 .
$$

De acuerdo con el resultado obtenido, la muestra corresponde a 165 enfermeras que laboran en el "Hospital Daniel Alcides Carrión". Para el muestreo se utilizó el muestreo aleatorio simple considerando la nómina proporcionada por el área de personal del hospital y una tabla de números aleatorios generada con el excel; para elegir a los 165 participantes. Por otra parte, según Hernandez, Fernandez y Baptista (2014), "La técnica de la encuesta recoge opiniones para obtener información fidedigna de la población" (p. 59). Por consiguiente, el presente estudio consideró como técnica a la encuesta para recoger información de las variables elegidas en el estudio. Asimismo, la temporalidad en el recojo de datos corresponde a un análisis de corte transversal realizado en un punto específico del tiempo. En tal sentido, los datos se recolectaron en tiempo único y en un solo momento para su respectivo análisis descriptivo e inferencial. Se hizo uso de un instrumento que mide la autoestima de autoría de Coopersmith conformada por 25 items de naturaleza dicotómica. Para realizar el análisis psicométrico se baremó la variable y las dimensiones en los niveles se muestran a continuación en la siguiente tabla:

\section{Tabla 3}

Consolidado de Niveles de las dimensiones de la variable (X) autoestima

\begin{tabular}{llrr}
\hline Niveles & $\begin{array}{l}\text { Dimesión 1 } \\
\text { En sí mismo general } \\
(13 \text { items })\end{array}$ & $\begin{array}{l}\text { Dimesión } 2 \\
\text { En el área social } \\
(6 \text { items })\end{array}$ & $\begin{array}{l}\text { Dimesión 3 } \\
\text { En el área del hogar } \\
(6 \text { items })\end{array}$ \\
\hline Alto & {$[39 ; 52]$} & {$[18 ; 24]$} & {$[18 ; 24]$} \\
Medio alto & {$[26 ; 38]$} & {$[12 ; 17]$} & {$[12 ; 17]$} \\
Medio bajo & {$[13 ; 25]$} & {$[6 ; 11]$} & {$[6 ; 11]$} \\
Bajo & {$[0 ; 12]$} & {$[0 ; 5]$} & {$[0 ; 5]$} \\
\hline
\end{tabular}

Fuente. test de autoestima 
El cuestionario de desempeño laboral consta 21 items de tipo ordinal y su baremos para la variable y sus dimensiones de acuerdo a los niveles se muestran en la siguiente tabla:

Tabla 4

Consolidado de Niveles de las dimensiones de la variable $(Y)$ desempeño laboral

\begin{tabular}{lcccc}
\hline Niveles & $\begin{array}{l}\text { Variable } \\
\text { Desepeño Laboral }\end{array}$ & $\begin{array}{l}\text { Dimesión 1 } \\
\text { Competencia } \\
\text { cognitiva }\left(Y_{1}\right)\end{array}$ & $\begin{array}{l}\text { Dimesión 2 } \\
\text { Competencias actitudinales y y } \\
\text { sociales }\left(Y_{2}\right)\end{array}$ & $\begin{array}{l}\text { Dimesión 3 } \\
\text { Competencia } \\
\text { técnica }\left(Y_{3}\right)\end{array}$ \\
\hline Muy bueno & {$[48 ; 63]$} & {$[19 ; 24]$} & {$[16 ; 21]$} & {$[15 ; 18]$} \\
Bueno & {$[32 ; 47]$} & {$[13 ; 18]$} & {$[11 ; 15]$} & {$[10 ; 14]$} \\
Regular & {$[16 ; 31]$} & {$[7 ; 12]$} & {$[6 ; 10]$} & {$[5 ; 9]$} \\
Malo & {$[0 ; 15]$} & {$[0 ; 6]$} & {$[0 ; 5]$} & {$[0 ; 4]$} \\
\hline
\end{tabular}

Fuente. Teoría de Chiavenato

De acuerdo con el análisis factorial la prueba de KMO (Kaiser-Meyer-Olkin) arrojó un coeficiente de 0,890 además la prueba de esfericidad de Bartlett mostró un valor de significancia de 0,000 , lo que indica que se puede hacer un análisis factorial en el número de variables consideradas. Por otro lado, el valor obtenido fue de $73,54 \%$, indicando su validez de constructo. Por tanto, el instrumento es válido.

\section{Tabla 5}

Distribución de la prueba KMO y Barlett de autoestima

\begin{tabular}{llr}
\hline \multicolumn{2}{l}{ Medida de adecuación muestral de Kaiser-Meyer-Olkin } & 0.890 \\
\hline \multirow{3}{*}{ Prueba de esfericidad de Barlett } & Chi-cuadrado aproximado & 1868,226 \\
& $g 1$ & 276 \\
& Sig. & 0,000 \\
\hline
\end{tabular}

Fuente. Base de datos

También se realizó la validación de constructo para el instrumento de desempeño laboral de manera factorial la naturaleza dimensional de estos indicadores, de este modo se calculó la correlación entre las escalas construidas al compararse las correlaciónes empíricas con las relaciónes teóricas determinadas entre los conceptos. De acuerdo con el análisis factorial la prueba de KMO (Kaiser-Meyer-Olkin) arrojó un coeficiente de 0,752 además la prueba de esfericidad de Bartlett mostró un valor de significancia de 0,000, lo que indica que se puede hacer un análisis factorial en el número de variables consideradas. Por otro lado, el valor obtenido fue de $83,391 \%$, indicando su validez de constructo. Por tanto, el instrumento fue válido. 


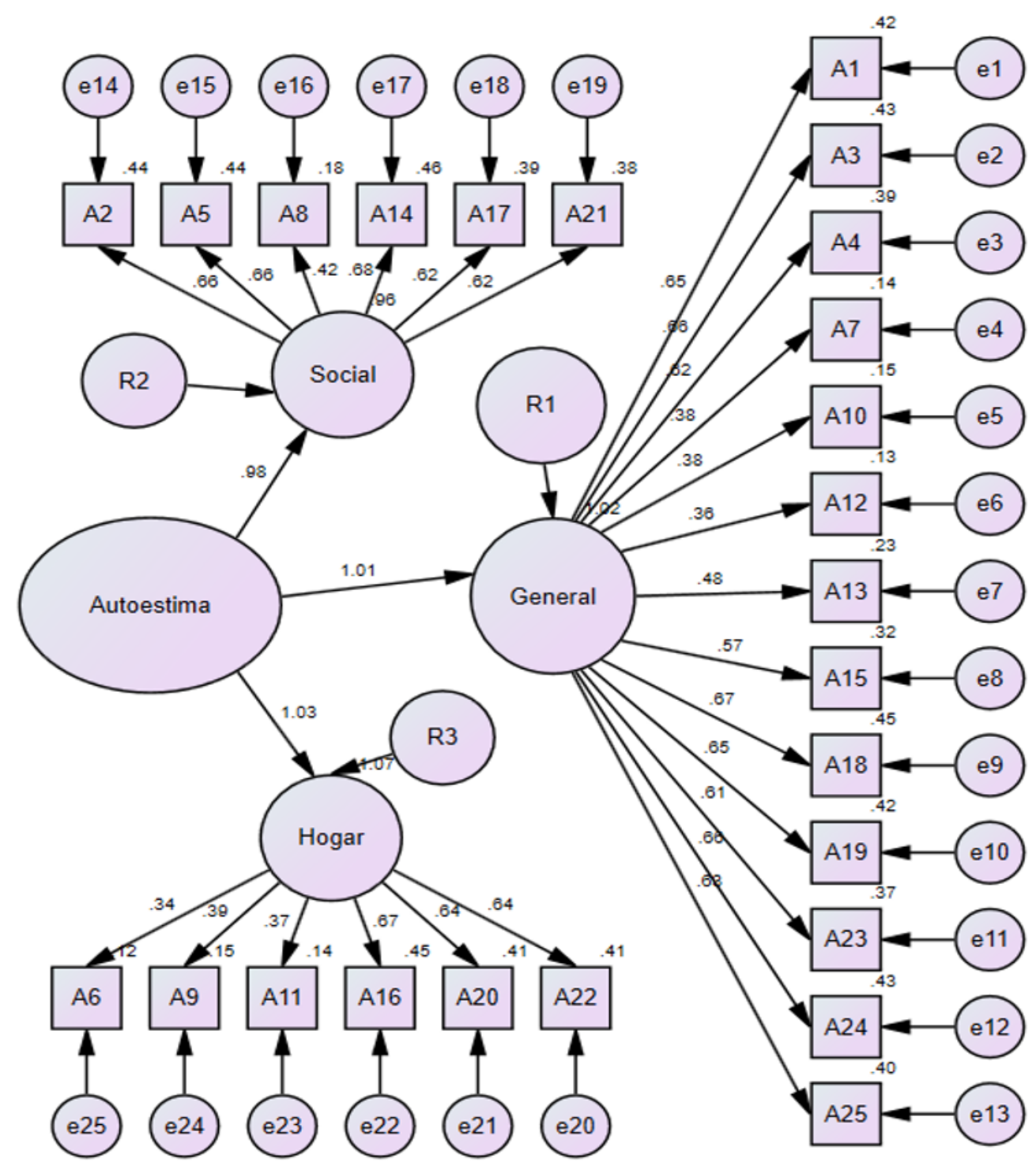

Figura 1. Diagrama del análisis factorial confirmatorio para la variable autoestima

Tabla 6

Distribución de la prueba KMO y Bartlett de desempeño laboral

\begin{tabular}{|c|c|c|}
\hline \multicolumn{3}{|c|}{$\begin{array}{c}\text { Prueba de KMO y Barlett } \\
\end{array}$} \\
\hline Medida Kaiser -Meyer-Olkin de a & ecuación de muestreo & 0,752 \\
\hline \multirow{3}{*}{ Prueba de esfericidad de Bartlett } & Aprox. Chi-cuadrado & 3691,483 \\
\hline & gl & 780 \\
\hline & Sig. & 0,000 \\
\hline
\end{tabular}

Fuente. Base de datos 


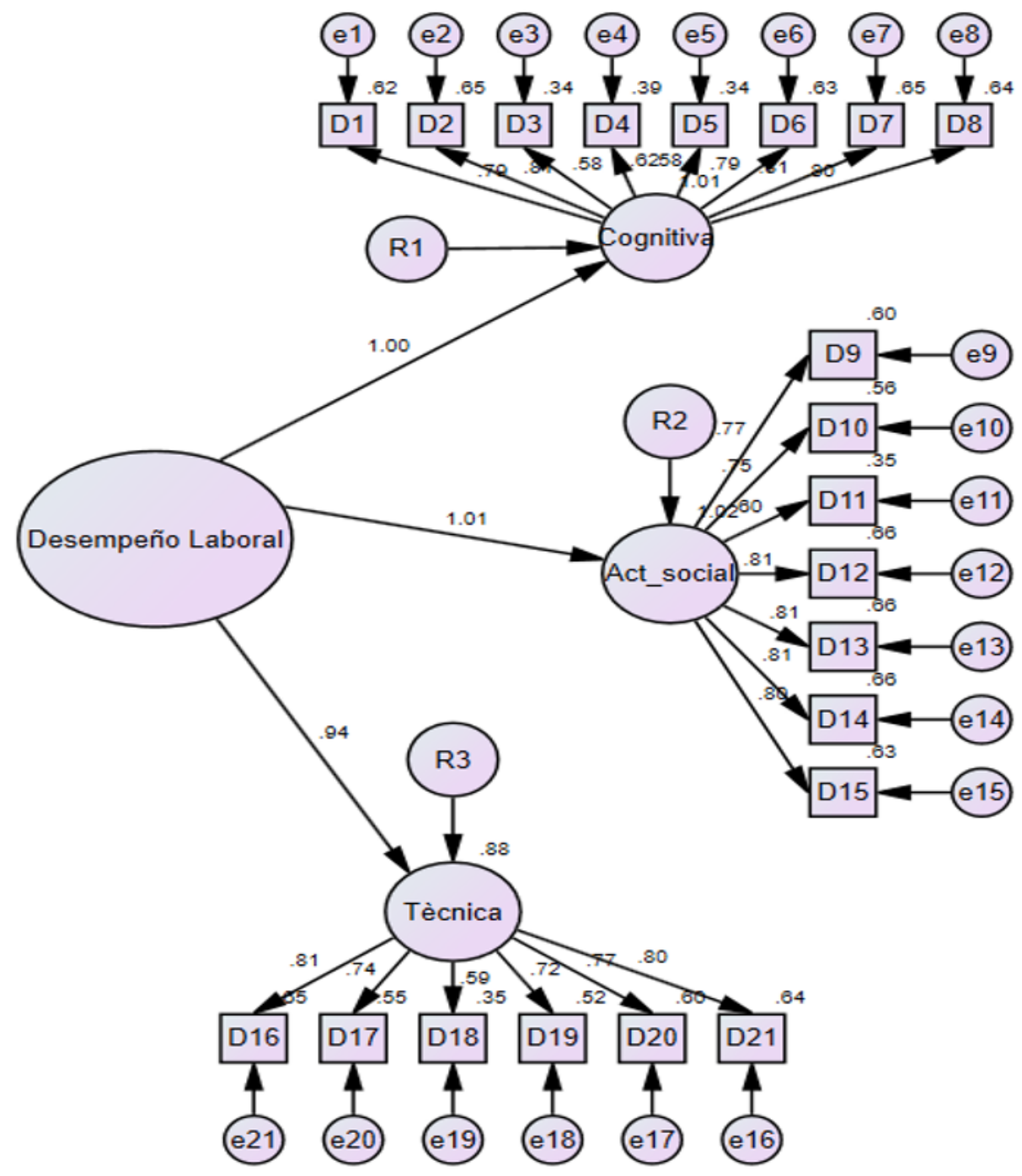

Figura 2. Diagrama del análisis factorial confirmatorio para la variable desempeño laboral

La consistencia interna del inventario aplicado para medir la variable $(X)$ : autoestima reportó a través del test retest 0,88 respecto a la confiabilidad en los 25 ítems evaluados. Asimismo, la consistencia interna del desempeño laboral $(Y)$ : fue analizado por los investigadores Diaz y Gaviria utilizando el estadístico de fiabilidad Alfa de Cronbach, alcanzando un valor de 0,913 en los 21 ítems evaluados.

Para el análisis estadistico se aplicó la prueba de independencia de variables mediante el estadístico Chi-cuadrado con una significancia del $5 \%$. Posteriormente, la relación entre las categorías de las variables y dimensiones se evaluo con el análisis de correspondencia simple, donde se presento la inercia y las contribuciones de las categorías en las dos dimensiones. Finalmente el mapa perceptual permite observar las correspondencias entre las categorias.

\section{Resultados}

La Tabla 7 muestra que el 82,4\% del personal de enfermería son mujeres; solo el 17,6\% son varones. El 76,4\% del personal de enfermería laboran en consulta externa; el 23,6\% trabajan en emergencia. La edad promedio fue de 38 años con una variación de 8 . Según el grupo etario 
el 21,8\% son jóvenes (mayores de 18 años pero menores de 30 ), el 74,6\% son adultos (de 30 años pero menores de 60) y 4 son adultos mayores (de 60 años a más).

\section{Tabla 7}

Resumen de los datos generales de las enfermeras del "Hospital Daniel Alcides Carrión", Callao $-2017$

\begin{tabular}{llllll}
\hline & Variables & $\mathbf{n}^{\circ}$ & $\%$ & Media & $\begin{array}{l}\text { Desviación } \\
\text { estándar }\end{array}$ \\
\hline Género & Femenino & 136 & 82,4 & & \\
& Masculino & 29 & 17,6 & & \\
Área & Consulta externa & 126 & 76,4 & & \\
Edad & Emergencia & 39 & 23,6 & & \\
& & & & 38 & \\
& Jóvenes & 36 & 21,8 & & \\
& Adulto & 123 & 74,6 & & \\
& Adulto mayor & 6 & 3,6 & & \\
\hline
\end{tabular}

Fuente. Base de datos

La Tabla 8 presenta las medidas de resumen de los puntajes totales de las variables y de cada dimensión. Los puntajes se obtuvieron considerando la suma de los códigos de respuesta de los ítems que conforman cada dimensión y variable. En el caso de la autoestima, las enfermeras alcanzan un promedio de 57 puntos con una variación de 29 , además los valores oscilan entre 8 a 100 puntos. Las 3 dimensiones que conforman la variable tienen promedios de 13 a 29 puntos. En cuanto al desempeño laboral, el promedio es 35 puntos con una variabilidad de 16, además los valores son de 8 a 58 puntos. Sus dimensiones tienen promedios de 10 a 13 puntos.

\section{Tabla 8}

Medidas de resumen de los puntajes de autoestima, desempeño laboral y las dimensiones en enfermeras del "Hospital Daniel Alcides Carrión", Callao 2017

\begin{tabular}{lcccc}
\hline & Media & $\begin{array}{c}\text { Desviación } \\
\text { Estándar }\end{array}$ & Mínimo & Máximo \\
\hline Autoestima & 57 & 29 & 8 & 100 \\
En sí mismo general & 29 & 16 & 0 & 52 \\
En el área social & 13 & 8 & 0 & 24 \\
En el área hogar & 14 & 7 & 0 & 24 \\
\hline Desempeño laboral & 35 & 16 & 8 & 58 \\
Competencia cognitiva & 13 & 6 & 2 & 22 \\
Competencias actitudinales y sociales & 12 & 6 & 1 & 20 \\
Competencia técnica & 10 & 5 & 1 & 18 \\
\hline
\end{tabular}

Fuente. Base de datos 
Considerando los baremos establecidos por los autores de ambos instrumentos, los puntajes fueron categorizados para su interpretación cualitativa. Estas se realizaron para cada variable y dimensión.

En la Tabla 9 se observa la distribución de las enfermeras según las categorías de autoestima. El 29,09\% de las enfermeras se encuentran en medio bajo al igual que la categoría medio alto. El 26, $06 \%$ de las enfermeras tienen autoestima alto. Solo el 15,76\% de las enfermeras están en la categoría bajo de autoestima. Además, se observa la distribución de las enfermeras según las dimensiones de autoestima. En el caso de la dimensión en sí mismo general, el 27,9\% de las enfermeras se ubican en la categoría medio alto, seguido del $26,7 \%$ en la categoría alto. En cuanto a la dimensión área social, la mayoría de enfermeras se encuentra en la categoría alto $(30,9 \%)$, contrario a esto, solo el $16,4 \%$ se encuentra en la categoría medio bajo. En la tercera dimensión (área hogar), el 33,3\% de las enfermeras se encuentra en la categoría medio alto, además, el $15,2 \%$ se ubica en la categoría bajo

\section{Tabla 9}

Distribución de enfermeras según autoestima y dimensiones

\begin{tabular}{llrr}
\hline Autoestima & $\mathbf{n}^{\circ}$ & $\mathbf{\%}$ & \\
\hline Bajo & 26 & 15,76 & \\
Medio bajo & 48 & 29,09 & \\
Medio alto & 48 & 29,09 & \\
Alto & 43 & 26,06 & \\
Total & 165 & 100,00 & \\
\hline Dimensiones & & $\mathbf{n}^{\circ}$ & $\mathbf{\%}$ \\
\hline \multirow{5}{*}{ En si mismo general } & Bajo & 32 & 19,4 \\
& Medio bajo & 43 & 26,1 \\
& Medio alto & 46 & 27,9 \\
& Alto & 44 & 26,7 \\
En el área social & Bajo & 37 & 22,4 \\
& Medio bajo & 27 & 16,4 \\
& Medio alto & 50 & 30,3 \\
& Alto & 51 & 30,9 \\
En el área hogar & Bajo & 25 & 15,2 \\
& Medio bajo & 33 & 20,0 \\
& Medio alto & 55 & 33,3 \\
& Alto & 52 & 31,5 \\
\hline
\end{tabular}

Fuente. Base de datos

En la Tabla 10 se observa la distribución de las enfermeras según las categorías del desempeño laboral, donde, el 40,6\% tiene muy buen desempeño, seguido del 31, $5 \%$ con desempeño regular. En porcentajes menores e iguales, se tiene las categorías malo y bueno con $13,9 \%$ cada uno. Además, se observa la distribución de las enfermeras según sus dimensiones; las categorías de la dimensión competencia cognitiva: donde, el 30,3\% tienen buen desempeño, seguido del 26,1\% con muy buen desempeño y el $20 \%$ de las tienen mal desempeño en la competencia cognitiva; en la dimensión competencias actitudinales y sociales: El 48,5\% tienen muy buen desempeño, otro porcentaje importante, se ubica en la categoría regular $(28,5 \%)$ y solo el $6,1 \%$ de las enfermeras tienen buen desempeño; en la dimensión competencia técnica: El 31,5\% tiene un desempeño regular, seguido del 29,1\% con buen desempeño, en porcentajes menores, el $20 \%$ tienen muy buen desempeño y el 19,4\% mal desempeño en la competencia técnica. 
Tabla 10

Distribución de enfermeras según desempeño laboral y dimensiones

\begin{tabular}{llrr}
\hline Autoestima & $\mathbf{n}^{\circ}$ & $\mathbf{\%}$ & \\
\hline Malo & 23 & 13,90 & \\
Regular & 52 & 31,50 & \\
Bueno & 23 & 13,90 & \\
Muy bueno & 67 & 40,60 & \\
Total & 165 & 100,00 & \\
\hline Dimensiones & Categorias & $\mathbf{n}^{\circ}$ & $\mathbf{\%}$ \\
\hline \multirow{2}{*}{ Competencia } & Malo & 33 & 20,00 \\
cognitiva & Regular & 39 & 23,60 \\
& Bueno & 50 & 30,30 \\
Competencia & Muy bueno & 43 & 26,10 \\
actitudinales y sociales & Malo & 28 & 17,00 \\
& Regular & 47 & 28,50 \\
& Bueno & 10 & 6,10 \\
Competencia cognitiva & Muy bueno & 80 & 48,50 \\
& Malo & 32 & 19,40 \\
& Regular & 52 & 31,50 \\
& Bueno & 48 & 29,10 \\
\hline
\end{tabular}

Fuente. Base de datos

El planteamiento de las hipótesis fueron: La autoestima se relacióna directa y significativamente con el desempeño laboral en las enfermeras del "Hospital Daniel Alcides Carrión", Callao - 2017. $H_{0}$ : La autoestima y el desempeño laboral son independientes.

$H_{1}$ : La autoestima y el desempeño laboral se relaciónan.

Tabla 11

Distribución de enfermeras según autoestima y desempeño laboral del "Hospital Daniel Alcides Carrión" - 2017

\begin{tabular}{|c|c|c|c|c|c|c|c|c|c|}
\hline & & & \multicolumn{4}{|c|}{ Desempeño laboral } & \multirow{2}{*}{ Total } & \multicolumn{2}{|c|}{$\begin{array}{c}\text { Prueba } \\
\text { Chi-Cuadrado }\end{array}$} \\
\hline & & & Malo & Regular & Bueno & $\begin{array}{l}\text { Muy } \\
\text { bueno }\end{array}$ & & $\chi^{2}$ & $p$ \\
\hline \multirow{8}{*}{ Autoestima } & \multirow{2}{*}{ Bajo } & $n^{\circ}$ & 11 & 4 & 5 & 6 & 26 & \multirow[t]{10}{*}{$33,898^{* *}$} & \multirow[t]{10}{*}{0,000} \\
\hline & & $\%$ & 42,40 & 15,40 & 19,20 & 23,10 & 100,00 & & \\
\hline & Medio & $n^{\circ}$ & 2 & 21 & 6 & 19 & 48 & & \\
\hline & bajo & $\%$ & 4,20 & 43,80 & 12,50 & 39,60 & 100,00 & & \\
\hline & Medio & $n^{\circ}$ & 2 & 19 & 7 & 20 & 48 & & \\
\hline & alto & $\%$ & 4,20 & 39,60 & 14,60 & 42,70 & 100,00 & & \\
\hline & & $n^{\circ}$ & 8 & 8 & 5 & 22 & 43 & & \\
\hline & Alto & $\%$ & 18,60 & 18,60 & 11,60 & 51,20 & 100,00 & & \\
\hline \multirow{2}{*}{\multicolumn{2}{|c|}{ Total }} & $n^{\circ}$ & 23 & 52 & 23 & 67 & 165 & & \\
\hline & & $\%$ & 13,90 & 31,50 & 13,90 & 40,60 & 100,00 & & \\
\hline
\end{tabular}


En la Tabla 11 y Figura 3, se observa que las enfermeras tienen baja autoestima, el 42, $3 \%$ tienen mal desempeño, el resto de categorías de desempeño presentan menor porcentaje. Contrario a ello, las enfermeras con categoría medio alto y alta autoestima, predomina el desempeño muy bueno con $41,7 \%$ y $51,2 \%$ respectivamente.

El estadístico Chi-cuadrado fue 33, 898 con $p=0,000<0,01$ entonces se rechaza la hipótesis nula, por lo tanto, "la autoestima se relacióna directa y significativamente con el desempeño laboral en las enfermeras del Hospital Daniel Alcides Carrión”, Callao - 2017.

El análisis de correspondencia simple entre las categorías de las variables procede debido a que las variables tienen relación significativa. En la Tabla 12 se observa que "la inercia es 0,205, del cual, el 99,6\% es explicado por las dos primeras dimensiones. El valor propio (autovalor) de la primera dimensión es 0,423 , además explica el $87,1 \%$ de la inercia".

\section{Tabla 12}

Auto valores, inercia total e incercia explicada por las dimensiones

\begin{tabular}{|c|c|c|c|c|c|c|}
\hline \multirow{3}{*}{ Dimensión } & \multirow{3}{*}{$\begin{array}{l}\text { Valor } \\
\text { propio }\end{array}$} & \multirow{3}{*}{ Inercia } & \multicolumn{2}{|c|}{ Proporción de inercia } & \multicolumn{2}{|c|}{$\begin{array}{c}\text { Confianza para el } \\
\text { valor propio }\end{array}$} \\
\hline & & & \multirow[t]{2}{*}{ Explicada } & \multirow[t]{2}{*}{ Acumulada } & \multirow{2}{*}{$\begin{array}{l}\text { Desviación } \\
\text { típica }\end{array}$} & Correlación \\
\hline & & & & & & 2 \\
\hline 1 & 0,423 & 0,179 & 0,871 & 0,871 & 0,077 & 0,091 \\
\hline 2 & 0,160 & 0,026 & 0,125 & 0,996 & 0,073 & \\
\hline 3 & 0,029 & 0,001 & 0,004 & 1,000 & & \\
\hline Total & & 0,205 & 1,000 & 1,000 & & \\
\hline
\end{tabular}

Fuente. Base de datos

En la Tabla 13 se observan las contribuciones de las categorías de la variable autoestima. La categoría bajo autoestima tiene una inercia de 0,119, además "explica el 63,6\% de la inercia en la primera dimensión y 20,5\% en la inercia de la segunda dimensión". Asimismo, "la categoría alto tiene inercia de 0,026, además explica el $68 \%$ de la inercia de la segunda dimensión".

Tabla 13

Contribuciones de las categorias de la variable autoestima

\begin{tabular}{|c|c|c|c|c|c|c|c|c|c|}
\hline \multirow{3}{*}{ Autoestima } & \multirow{3}{*}{ Masa } & \multicolumn{2}{|c|}{$\begin{array}{l}\text { Puntuación en } \\
\text { la dimensión }\end{array}$} & \multirow{3}{*}{ Inercia } & \multicolumn{5}{|c|}{ Contribución } \\
\hline & & \multirow[t]{2}{*}{1} & \multirow[t]{2}{*}{2} & & \multicolumn{2}{|c|}{$\begin{array}{l}\text { De los puntos a } \\
\text { la inercia de la } \\
\text { dimensión }\end{array}$} & \multicolumn{3}{|c|}{$\begin{array}{l}\text { De la dimensión a la } \\
\text { inercia del punto }\end{array}$} \\
\hline & & & & & 1 & 2 & 1 & 2 & Total \\
\hline Bajo & 0,158 & $-1,307$ & 0,457 & 0,119 & 0,636 & 0,205 & 0,956 & 0,044 & 1,000 \\
\hline Medio bajo & 0,291 & 0,505 & 0,230 & 0,034 & 0,176 & 0,096 & 0,917 & 0,072 & 0,989 \\
\hline Medio alto & 0,291 & 0,452 & 0,102 & 0,026 & 0,141 & 0,019 & 0,963 & 0,019 & 0,982 \\
\hline Alto & 0,261 & $-0,279$ & $-0,646$ & 0,026 & 0,048 & 0,680 & 0,329 & 0,671 & 1,000 \\
\hline Total & 1,000 & & & 0,205 & 1,000 & 1,000 & & & \\
\hline
\end{tabular}

Fuente. Base de datos 
En la Tabla 14 se observa "la contribución de las categorías del desempeño laboral en la inercia de cada dimensión biespacial". Entre las categorías, la que tiene mayor inercia es el desempeño malo con 0,135 ; además, "esta categoría explica el $75,1 \%$ de la inercia de la primera dimensión. En cambio, la inercia de la dimensión 2 es explicada en su mayoría por la categoría muy bueno con un $56,8 \%$ ".

\section{Tabla 14}

Contribuciones de las categorias de la variable desempeño laboral

\begin{tabular}{|c|c|c|c|c|c|c|c|c|c|}
\hline \multirow{3}{*}{$\begin{array}{l}\text { Desempeño } \\
\text { laboral }\end{array}$} & \multirow{3}{*}{ Masa } & \multicolumn{2}{|c|}{$\begin{array}{l}\text { Puntuación en } \\
\text { la dimensión }\end{array}$} & \multirow{3}{*}{ Inercia } & \multicolumn{5}{|c|}{ Contribución } \\
\hline & & \multirow[t]{2}{*}{1} & \multirow[t]{2}{*}{2} & & \multicolumn{2}{|c|}{$\begin{array}{l}\text { De los puntos a } \\
\text { la inercia de la } \\
\text { dimensión }\end{array}$} & \multicolumn{3}{|c|}{$\begin{array}{l}\text { De la dimensión a la } \\
\text { inercia del punto }\end{array}$} \\
\hline & & & & & 1 & 2 & 1 & 2 & Total \\
\hline Malo & 0,139 & $-1,510$ & 0,141 & 0,135 & 0,751 & 0,017 & 0,996 & 0,003 & 0,999 \\
\hline Regular & 0,315 & 0,534 & 0,410 & 0,047 & 0,212 & 0,331 & 0,815 & 0,182 & 0,997 \\
\hline Bueno & 0,139 & $-1,178$ & 0,311 & 0,005 & 0.010 & 0.084 & 0.398 & 0.460 & 0.858 \\
\hline Muy bueno & 0,406 & 0,165 & $-0,473$ & 0,019 & 0,026 & 0,568 & 0,243 & 0,757 & 1,000 \\
\hline Total & 1,000 & & & 0,205 & 1,000 & 1,000 & & & \\
\hline
\end{tabular}

Fuente. Base de datos

En el mapa perceptual (Figura 3) se observa "las correspondencias de las categorías de ambas variables". Se puede afirmar que, "el bajo autoestima se corresponde con el mal desempeño laboral de las enfermeras; además, la autoestima alto se corresponde con el muy buen desempeño laboral".
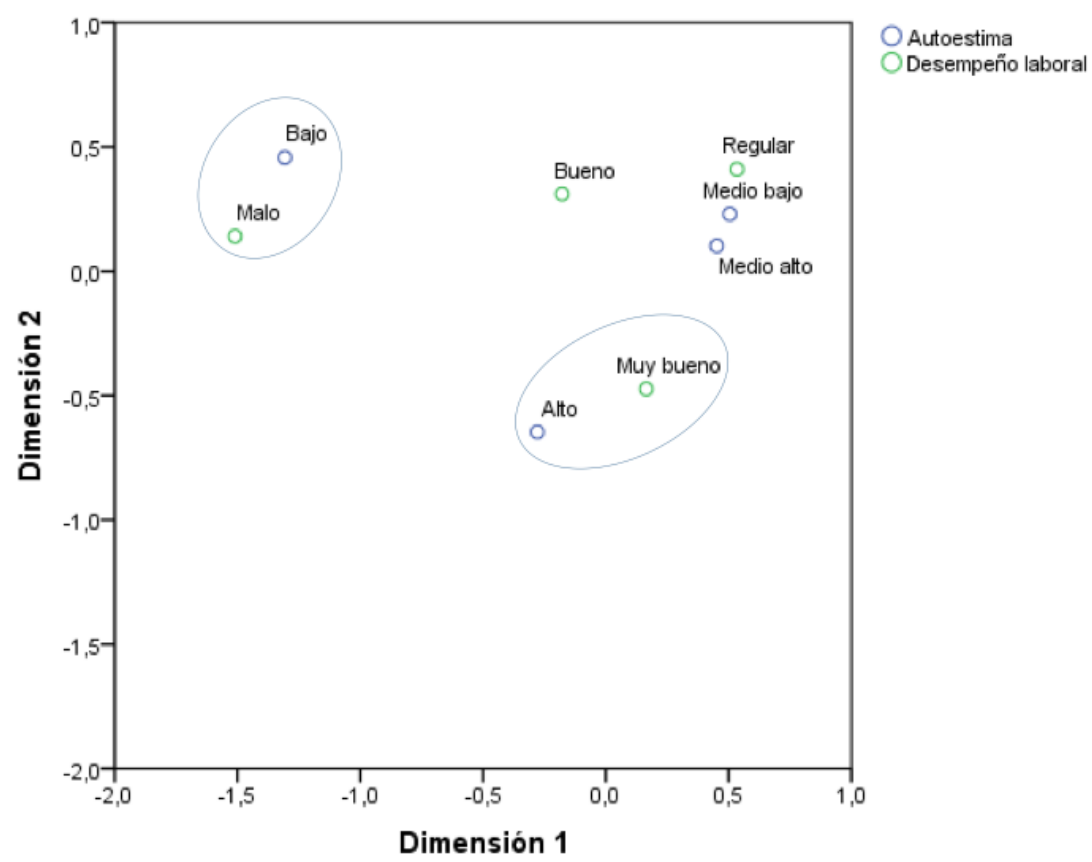

Figura 3: Mapa perceptual de autoestima y desempeño laboral en enfermeras del "Hopital Daniel Alcides Carrión”, Callao - 2017. 


\section{Discusión}

La autoestima es una variable muy importante en el mundo laboral generando un estado ideal para el crecimiento en las organizaciones; esta se puede manifestar en buena interacción con los demás compañeros y su desempeño laboral. En el caso del objetivo general, en los resultados del presente estudio se encontró que existe relación significativa entre el autoestima y el desempeño laboral de las enfermeras $\left(\chi^{2}=33,898 p<0,01\right)$, además la categoría bajo autoestima se corresponde con el mal desempeño laboral; asimismo, el alto autoestima-se corresponde con el muy buen desempeño laboral. Al respecto, Machuca (2016) en su estudio señala que la disposición en el trabajo permite un buen desempeño laboral; dicha disposición se presenta en mayor o menor medida de acuerdo al autoestima de la enfermera. En la misma línea, Raynaga (2015) encontró relación significativa entre motivación y desempeño laboral, dicha motivación se fortalece con una buena autoestima.

\section{Conclusiones}

- La autoestima se relacióna significativamente y de manera directa con el desempeño laboral en las enfermeras del "Hospital Daniel Alcides Carrión", Callao - 2017. La enfermera con bajo autoestima se corresponde principalmente con el mal desempeño laboral.

- La autoestima de las enfermeras estaba en su mayoría en un nivel medio. Entre las dimensiones de la autoestima estaban mejor en la dimensión hogar pero estaban un poco mal en la dimensión si mismo.

- El desempeño de las enfermeras se encontró en su mayoría muy bueno. Entre las dimensiones la mejor fue las competencias actitudinales y sociales pero la competencia cognitiva es donde tuvieron falencia

\section{Recomendaciones}

- En futuros estudios se sugiere incluir otras variables que expliquen el desempeño laboral.

- Incrementar el tamaño de muestra para futuros estudios e incluir estudios de tipo explicativo.

- Al establecimiento de salud se sugiere adoptar los resultados como política de desarrollo profesional de las enfermeras.

- Mejorar la relación entre autoestima y la competencia técnica de las enfermeras.

\section{Referencias bibliográficas}

[1] Becerra, C. (2017). Influencia del clima organizacional en el desempeño laboral(Tesis de pregado). Recuperado de: http://tesis.ucsm.edu.pe/repositorio/bitstream/handle/UCSM /6055/53.0858.AE.pdf?sequence=1\&isAllowed=y

[2] Bernaola, L. (2008). Estudio correlaciónal entre estilos de crianza e indefensión aprendida en estudiantes del quinto y sexto grado de primaria de la Institución Educativa № 2013 "Asociación Policial" S.M.P.-2007. (Tesis de Licenciatura). Universidad Mayor de San Marcos, Lima, Perú.

[3] Bolinches, A. (2015). El secreto de la autoestima. España: ediciones BSA. 
[4] Chiavenato, I. (2007). Administración de recursos humanos. Colombia: Mc GrawHill/Interamericana.

[5] Hernández, R., Fernández, C. y Baptista, P. (2014). Metodología de la investigación. México: McGraw. Hill/interamericana Editores S.A.

[6] Lladó, D., Sánchez, L., y Navarro, M. (2013). Competencias profesionales y empleabilidad en el contexto de la flexibilidad laboral. Estados Unidos de América: Palibrio.

[7] Machuca, S. (2016). Determinantes del trabajo en el desempeño laboral de los licenciados de enfermería en el hospital I ESSALUD- Tingo María. (Tesis de maestría). Universidad de Huánuco, Huánuco, Perú.

[8] Mejía, Y. (2012). Evaluación del desempeño con enfoque en las competencias laborales. Estudio realizado con personal de servicio de un hospital en la ciudad de Quetzaltenango. (Tesis doctoral). Universidad Rafael Landívar, Guatemala.

[9] Mireyna, R. (2012). Motivación, satisfacción y desempeño laboral en profesional es de enfermería. (Tesis doctoral). Universidad de Deusto, Bilbao, España.

[10] Organización Mundial de la Salud (2014). Protección de la salud de los trabajadores. Recuperado de: http://www.who.int/mediacentre/factsheets/fs389/es/

[11] Reynaga, Y. (2015). Motivación y desempeño laboral del personal de enfermería en el Hospital Hugo Pesce Pescetto de Andahuaylas, 2015. (Tesis de doctoral). Universidad Nacional José María Arguedas, Andahuaylas, Perú.

[12] Rodríguez, I. (2013). La autoestima y su relación con el aprendizaje. Recuperado de: repositorio.une.edu.pe/bitstream/handle/UNE/707/T0251 0722928_ T.pdf.

[13] Soto, I. (2015). La tesis en 4 pasos. Perú: Nuevo Milenio.

[14] Tamayo, M. (2012). El proceso de la investigación científica. México: Limusa.

[15] Trujillano, J. (2014). El enfoque en competencias y la mejora en educación. Ra Ximhai, 10(5), 307-322. Recuperado de: http://www.redalyc.org/pdf/461/46132134026.pdf.

[16] Vara, A. (2015). 7 pasos para elaborar una tesis. Perú: Macro 